\title{
Evaluación clínica del adaptado de retenedores directos de dentaduras parciales removibles a extensión distal
}

\section{Clinical evaluation of direct retainer adaptation in distal extension removable partial dentures}

\author{
Sánchez Ysmayel AE*, Villarroel Dorrego $M^{* *}$
}

\begin{abstract}
RESUMEN
Introducción: El propósito del presente estudio fue evaluar clínicamente el adaptado de los retenedores directos en Dentaduras Parciales Removibles (DPR) a extensión distal y determinar si el diseño del retenedor empleado impactaba sobre el adaptado de la misma.

Materiales y métodos: Fueron evaluados 84 sujetos a los cuales le fueron instaladas DPR. La evaluación del adaptado incluyó la utilización de un material de silicona fluida como sustancia detectora de contacto y con el uso de un calibrador digital se determinó la distancia del tope sobre el descanso. Este espacio fue calculado en mm y la media \pm desviación estándar determinada. La relación entre el adaptado de los elementos del retenedor directo y el tipo de retenedor empleado se comparó aplicando las pruebas de chi cuadrado y $t$ de Student considerando diferencias estadísticamente significativas cuando $p<0,05$.

Resultados: Se evaluaron 168 retenedores directos, observando que solo el 9\% contactaba en el centro del tope. El espacio promedio fue de 0,1892 $\pm 0,25216 \mathrm{~mm}$ y no se observó diferencia $(p=0,261)$ según el tipo de retenedor directo empleado. En los elementos retentivos y estabilizadores se observó que el contacto ocurría mayormente en la parte terminal o extremo y adaptado de estos elementos varió según el tipo de retenedor directo empleado $(p=0,0001)$.

Discusión: En las DPR evaluadas el adaptado del retenedor directo al diente fue deficiente y el ajuste de los elementos retentivos y estabilizadores fue directamente asociado al diseño del retenedor. El uso de DPR es una solución práctica y de bajo costo para la rehabilitación protésica de gran parte de la población, sin embargo su cuidadoso diseño y el correcto adaptado de los retenedores deben considerarse estrictamente para garantizar la salud bucal del individuo.
\end{abstract}

Palabras clave: Ajuste, adaptado, retenedores, dentaduras parciales removibles, prótesis.

\section{SUMMARY}

Introduction: The purpose of this study was to clinically evaluate the adaptation of direct retainers used in distal extension Removable Partial Dentures (RPD) and to determine the relationship between the retainer design and the adjustment of this element.

Materials $\mathcal{E}$ methods: 84 subjects recently rehabilitated with RPD were included in the study. The evaluation process included the use of a fluid silicone material as a detection substance in addition to a digital gauge to determinate the distance of the rest upon the abutment teeth. Space was calculated in $\mathrm{mm}$ and mean \pm standard deviation determined. Adjustment of the retainers was compared using chi $^{2}$ and $t$ Student tests. P values $<0,05$ were considered statistically significant.

Results: A total of 168 direct retainers were evaluated and only $9 \%$ contact in the center of the rest was observed. Average of space was 0,1892 $\pm 0,25216 \mathrm{~mm}$ and no difference was observed when type of retainers

* Profesor Asociado del Departamento de Prótesis. Especialista en Prostodoncia. Universidad Central de Venezuela.

** Profesora Asociado del Instituto de Investigaciones Odontológicas. Universidad Central de Venezuela. 
was compared $(p=0,261)$. On retentive and stabilizing elements of the direct retainers it was noted that contact occurs mostly on its terminal part, however, adjustment of those elements varied depending on the design of direct retainer used $(p=0,0001)$.

Discussion: Direct retainer adjustment of RPD evaluated was deficient and adaptation of retentive and stabilizing elements was directly dependant on direct retainer design. RPD are a low cost and practical solution for population dental rehabilitation, nonetheless, correct prosthetic plan and strict adaptation of the denture are required to warrant an oral healthy status.

Key words: Adjustment, retainer, removable partial dentures, dental prosthesis.

Fecha de recepción: Enero de 2011.

Aceptado para publicación: Marzo de 2011.

Sánchez Ysmayel AE, Villarroel Dorrego M. Evaluación clínica del adaptado de retenedores directos de dentaduras parciales removibles a extensión distal. Av. Odontoestomatol 2012; 28 (3): 151-158.

\section{INTRODUCCIÓN}

El aspecto más significativo del tratamiento con Dentaduras Parciales Removibles (DPR) es la cuidadosa planificación, ejecución de la preparación de la boca y su reproducción exacta a través del proceso de fabricación. Así por ejemplo, descansos correctamente preparados y adaptados a sus topes dirigirán las fuerzas de masticación para que los dientes y la estructura produzcan en conjunto un equilibrio y distribución de las fuerzas lo que contribuye a la mayor longevidad de las estructuras bucales remanentes y la restauración (1).

En este sentido, el estudio realizado por Frank et al (2) donde se aplican algunos estándares establecidos por la Academia Americana de Prostodoncia para la evaluación de la forma del apoyo, la extensión de la base, la ubicación de los apoyos en dientes adyacentes a la extensión distal y ajuste de la estructura, se comprobó que las probabilidades de daño periodontal aumentan cuando alguno de estos factores no está presente.

Los errores técnicos en la fabricación, la deformación de las impresiones y los diseños inapropiados, causan un exceso o una fuerza de retención deficiente. Los métodos de ajuste negligentes para incrementar o disminuir la retención a través del desgaste, el pulido o el doblez de los brazos de los ganchos pueden llevar a la inestabilidad de la DPR, al daño de los tejidos circundantes y a la fractura del gancho. La retención excesiva se puede reducir a través del método apropiado lo cual dependerá de la causa, mientras que la retención deficiente se puede incrementar temporalmente, pero decaerá de nuevo (3).

En estudio previo se evaluó la calidad de las estructuras metálicas desde el punto de vista técnico (sobre los modelos de trabajo), y se evidenció que menos del $50 \%$ de los retenedores evaluados cumplen con los requisitos en cuanto a la ubicación de los elementos retentivos, estabilizadores y ubicación de la punta retentiva en la calibración. Sin embargo la evaluación de los elementos de soporte de estos retenedores mostró que $86 \%$ se encontraban de forma aceptable cuando se evaluó extensión y ubicación (4). En este sentido llama la atención que, en la evaluación del ajuste clínico de los retenedores, Dunham et al. (5) observaron que el 76\% de los apoyos no presentan contacto en la superficie interna.

Se ha observado que el retenedor combinado colado forjado seguido por el retenedor tipo RPA son los utilizados con mayor frecuencia en la solución de los casos de DPR a extensión distal y se ha asociado esta situación a su efecto de rompefuerza $(4,6,7)$. Un brazo retentivo de alambre forjado flexiona en todas direcciones con más facilidad que los brazos colados en media caña, por tanto, pueden disipar con más eficiencia las tensiones que se trasmiten a los pilares pero presentan desventajas relacionas con la facilidad con la que se puede distorsionar (8), por lo que es muy probable que su adaptado y funcionamiento se pueda ver comprometido. 
De lo planteado se desprende la importancia de realizar una investigación con el propósito de evaluar clínicamente el adaptado de cada componente de los retenedores directos y determinar la relación del ajuste con el tipo de retenedor directo empleado en DPR a extensión distal.

\section{MATERIALES Y MÉTODOS}

Se presenta un estudio descriptivo de corte transversal, con diseño de una investigación de campo, donde la población estudiada la constituyen 84 sujetos tratados protésicamente en el pregrado de la Facultad de Odontología de la Universidad Central de Venezuela dados de alta en el periodo mayo-julio de 2008, que aceptaron ser evaluados de acuerdo a Consentimiento Informado aprobado por la Comisión de Bioética de la Facultad de Odontología y cuyo tratamien- to incluyó la instalación de una DPR Clase I o II de Kennedy mandibular. Fueron consideradas estas situaciones por ser las más frecuentes y comúnmente asociadas a complicaciones según estudios previos (2).

Para la evaluación del adaptado de los elementos del gancho se empleó el mismo procedimiento utilizado y validado por Dunham et al. (5), quienes utilizaron un material de polivinilsiloxano (PVS) como sustancia detectora. En este caso se empleó el material de impresión PVS en consistencia fluida President (ColteneR Art N4667 Lot 0121724). El procedimiento consistió en inyectar el material PVS alrededor de toda la superficie interna de la estructura de los ganchos de la DPR incluyendo conectores menores, posteriormente la DPR fue firmemente insertada en boca en posición con presión digital hasta asentar completamente la prótesis, una vez de polimerizado el material de impresión se retiró la DPR para la inspección (Fig. 1)
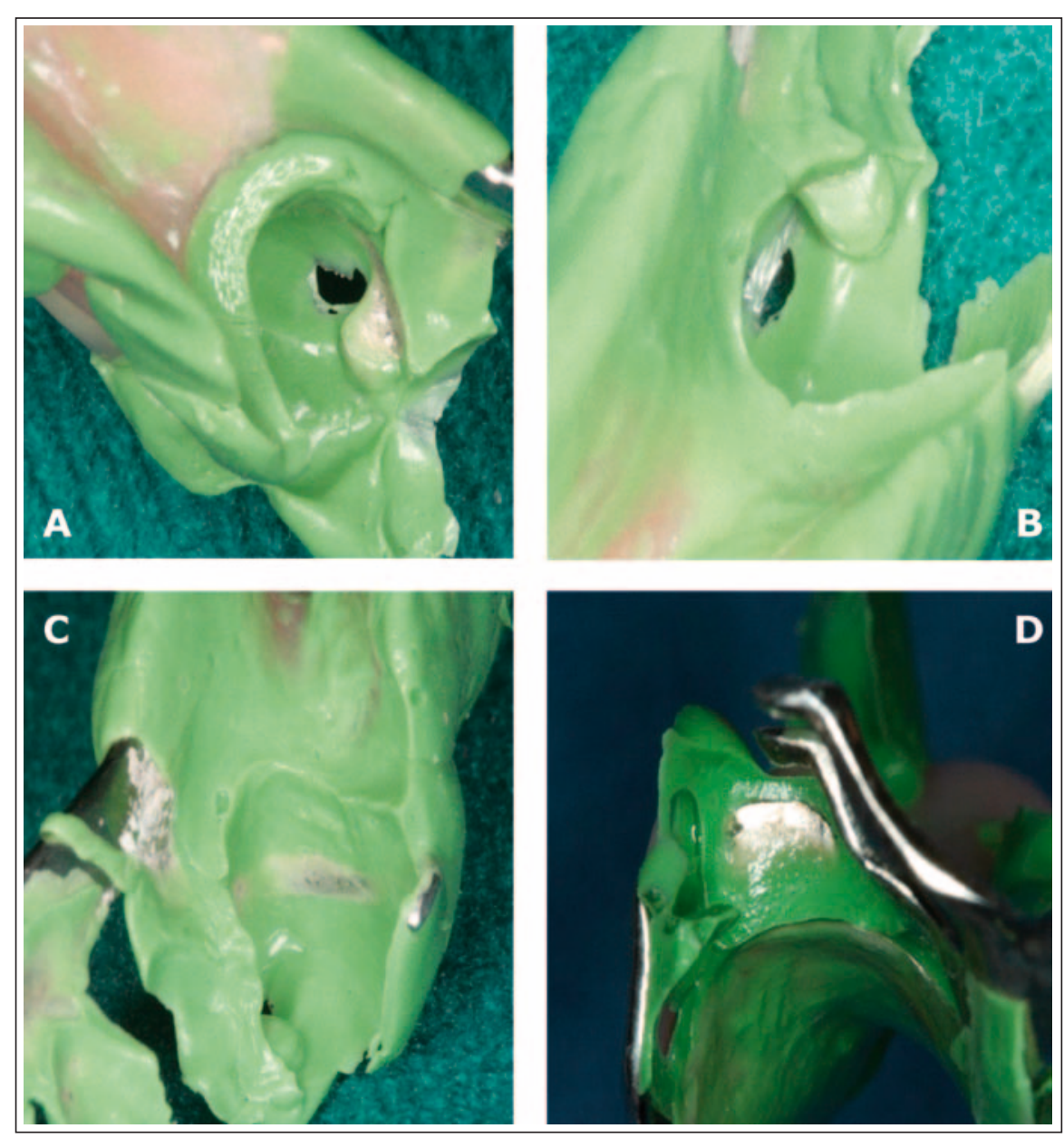

Fig. 1. Procedimiento del evaluación del adaptado.

A: Tope con contacto en el centro.

B: Tope sin contacto en el centro.

C y D: Evaluación del elemento retentivo y estabilizador. 
La evaluación fue realizada siempre por el mismo operador, valorando el contacto en los siguientes elementos: Elemento retentivo y elemento estabilizador donde a su vez se observaron tres zonas (en la unión con el conector menor, en la zona media y en el extremo); el tope se evaluó en tres zonas (centro, bordes y en la unión con el conector). En los casos donde el diseño de retenedor empleado era RPI o alguna de sus modificaciones se consideró la placa proximal como elemento estabilizador. Todos estos datos y el tipo o diseño de retenedor directo (gancho) empleado se registraron en un instrumento de recolección de datos.

Especímenes que no mostraban contacto en el centro del tope se evaluaron para calcular el espacio entre el metal y el diente. El registro de material de PVS fue cuidadosamente retirado de la estructura del gancho y con calibrador digital (Neiko ProQuality) con una sensibilidad de diezmilésimas de mm (micrómetros) (Fig. 2). Se determinó y registró la medida de espesor en la parte correspondiente al centro del tope.

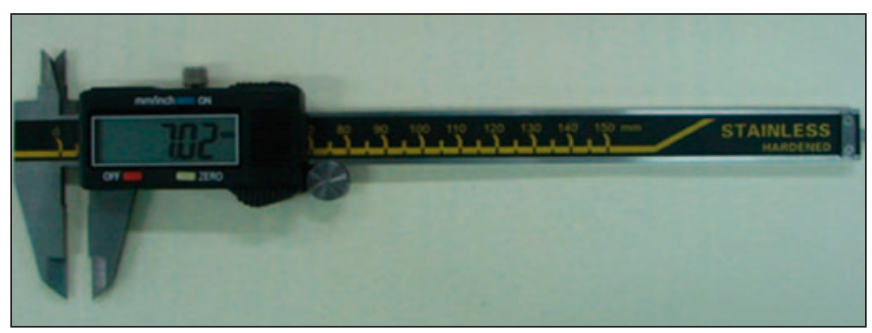

Fig. 2. Calibrador digital usado para medir el espacio entre el tope y el descanso del diente.

La data recolectada se ordenó para el análisis estadístico descriptivo (distribución de frecuencias) de las variables estudiadas, para lo cual se empleó el programa estadístico SPSS versión 17.0. El espacio entre el centro del tope y el descanso fue calculado en mm y la media \pm desviación estándar calculadas. La relación entre el adaptado de los diferentes elementos del retenedor directo y el tipo de retenedor empleado, se estudió aplicando la prueba de chi cuadrado y $t$ de Student (o estadístico exacto de Fisher cuando era necesario) considerando un nivel de significancia de $\mathrm{p}<0,05$.

\section{RESULTADOS}

Fueron evaluados 168 retenedores directos. El tipo de retenedor directo empleado con mayor frecuencia fue el combinado Colado-Forjado, seguido del retenedor RPA (Figura 3). En la evaluación del adaptado de los diferentes elementos de los retenedores directos mediante la identificación de la presencia o no de contacto, se observó que el contacto del tope sobre el descanso ocurrió mayormente en el borde $(45 \%)$ y solo el $9 \%$ contactó en el centro del tope. En los elementos retentivos y estabilizadores se observó el contacto mayormente en la parte terminal o extremo, $64 \%$ y $47 \%$ respectivamente, mientras que en la unión de estos elementos con el conector menor se evidenció el porcentaje menor de contacto (Figura 4).

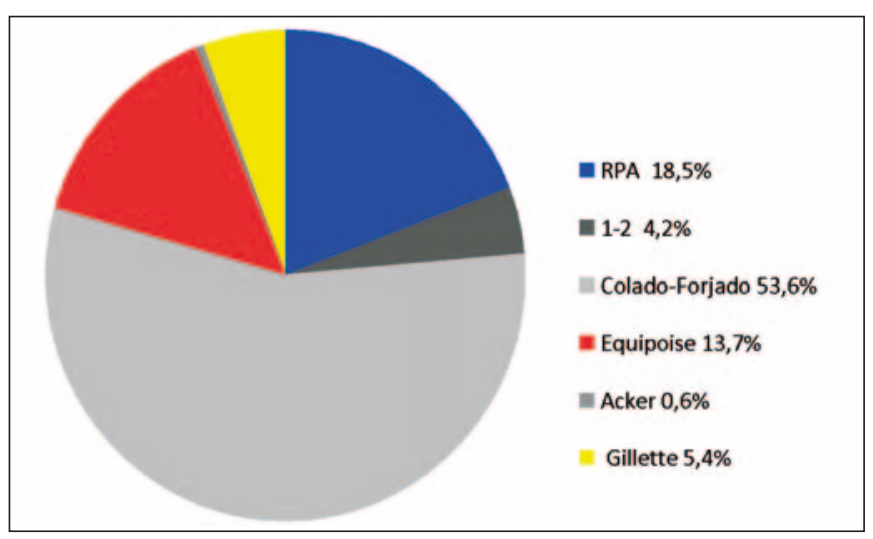

Fig. 3. Tipo retenedor directo.

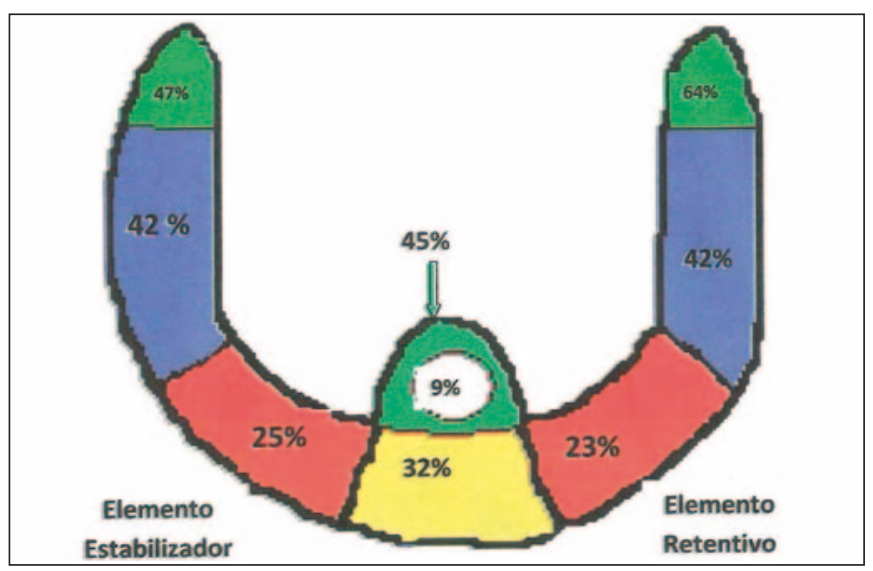

Fig. 4. Presencia de contacto en las áreas evaluadas de los Retenedores Directos empleados en DPR a extensión distal. 
En los casos donde no se observó contacto del centro del tope sobre el descanso, el espacio en promedio fue de $0,1892 \mathrm{~mm} \pm 0,25216$. Cuando se comparó la presencia o no de contacto según el tipo de retenedor directo empleado (Tabla 1), no se observó una diferencia estadísticamente no significativa $(\mathrm{p}=$ $0,261)$.

En la evaluación del adaptado del elemento retentivo y estabilizador según el tipo de retenedor directo empleado (tablas 2 y 3 ) se observó que existe diferencia estadísticamente significativa según los diferentes tipos de retenedores directos ( $p=0,0001)$.

Sin embargo, cuando se comparan solo los retenedores directos empleados con mayor frecuencia (Colado-Forjado y RPA), se observa que en la evaluación del adaptado elemento retentivo no existe diferencia estadísticamente significativa $(p=0,062)$. Igualmente en la evaluación del adaptado del elemento esta-

TABLA 1.- DISTRIBUCIÓN DE LOS RETENEDORES DIRECTOS UTILIZADOS EN DPR A EXTENSIÓN DISTAL SEGÚN EL TIPO Y LA PRESENCIA DE CONTACTO DEL CENTRO DEL TOPE CON EL DIENTE PILAR

\begin{tabular}{|l|c|c|c|c|c|c|c|c|}
\hline \multirow{2}{*}{$\begin{array}{l}\text { Contacto del centro del } \\
\text { tope con el diente pilar }\end{array}$} & \multicolumn{7}{|c|}{ Tipo de retenedor directo* } & \multirow{2}{*}{ Total } \\
\cline { 2 - 10 }$n$ & RPI & RPA & $\mathbf{1 - 2}$ & Colado-Forjado & Equipoise & Acker & Gillette & \\
\hline No hay contacto & 5 & 26 & 7 & 83 & 22 & 1 & 9 & 153 \\
\hline Si hay contacto & 2 & 5 & 0 & 7 & 1 & 0 & 0 & 15 \\
\hline Total & 7 & 31 & 7 & 90 & 23 & 1 & 9 & 168 \\
\hline$* \mathrm{p}=0,261$ & \multicolumn{7}{|c|}{} \\
\hline
\end{tabular}

\section{TABLA 2.- DISTRIBUCIÓN DE LOS DE RETENEDORES DIRECTOS EMPLEADOS EN DPR A EXTENSIÓN DISTAL SEGÚN EL TIPO Y LA FORMA DE CONTACTO DEL ELEMENTO RETENTIVO CON EL DIENTE PILAR}

\begin{tabular}{|l|c|c|c|c|c|c|c|r|r|}
\hline & \multicolumn{7}{|c|}{ Contacto del elemento retentivo con diente pilar* } & \\
\cline { 2 - 11 } $\begin{array}{l}\text { Tipo } \\
\text { retenedor } \\
\text { directo }\end{array}$ & $\begin{array}{c}\text { No hay } \\
\text { contacto }\end{array}$ & $\begin{array}{c}\text { Unión } \\
\text { con el } \\
\text { conector } \\
\text { menor }\end{array}$ & $\begin{array}{l}\text { Zona } \\
\text { media }\end{array}$ & Extremo & $\begin{array}{c}\text { Unión con } \\
\text { conector } \\
\text { menor y } \\
\text { zona } \\
\text { media }\end{array}$ & $\begin{array}{c}\text { Unión } \\
\text { con } \\
\text { conector } \\
\text { menor y } \\
\text { extremo }\end{array}$ & $\begin{array}{c}\text { Zona } \\
\text { media y } \\
\text { extremo }\end{array}$ & $\begin{array}{c}\text { Unión con } \\
\text { conector } \\
\text { menor, } \\
\text { zona media } \\
\text { y extremo }\end{array}$ & Total \\
\hline RPI & 0 & 0 & 1 & 4 & 0 & 1 & 1 & 0 & 7 \\
\hline RPA & 4 & 1 & 3 & 8 & 0 & 3 & 7 & 5 & 31 \\
\hline $1-2$ & 1 & 0 & 1 & 0 & 1 & 1 & 0 & 3 & 7 \\
\hline Colado-forjado & 10 & 5 & 16 & 33 & 3 & 2 & 12 & 9 & 90 \\
\hline Equipoise & 3 & 1 & 0 & 11 & 0 & 0 & 5 & 3 & 23 \\
\hline Acker & 1 & 0 & 0 & 0 & 0 & 0 & 0 & 0 & 1 \\
\hline Gillette & 9 & 0 & 0 & 0 & 0 & 0 & 0 & 0 & 9 \\
\hline Total & 28 & 7 & 21 & 56 & 4 & 7 & 25 & 20 & 168 \\
\hline$*$ p=0,0001 & & & & & & & & \\
\hline
\end{tabular}




\section{TABLA 3.- DISTRIBUCIÓN DE LOS DE RETENEDORES DIRECTOS EMPLEADOS EN DPR A EXTENSIÓN DISTAL SEGÚN EL TIPO Y LA FORMA DE CONTACTO DEL ELEMENTO RETENTIVO CON EL DIENTE PILAR}

\begin{tabular}{|l|c|c|c|c|c|c|c|c|r|}
\hline \multirow{2}{*}{$\begin{array}{l}\text { Tipo } \\
\text { retenedor } \\
\text { directo }\end{array}$} & $\begin{array}{c}\text { No hay } \\
\text { contacto }\end{array}$ & $\begin{array}{c}\text { Unión } \\
\text { con el } \\
\text { conector } \\
\text { menor }\end{array}$ & $\begin{array}{l}\text { Zona } \\
\text { media }\end{array}$ & Extremo & $\begin{array}{c}\text { Unión con } \\
\text { conector } \\
\text { menor y } \\
\text { zona } \\
\text { media }\end{array}$ & $\begin{array}{c}\text { Unión } \\
\text { con } \\
\text { conector } \\
\text { menor y } \\
\text { extremo }\end{array}$ & $\begin{array}{c}\text { Zona } \\
\text { media y } \\
\text { extremo }\end{array}$ & $\begin{array}{c}\text { Unión con } \\
\text { conector } \\
\text { menor, } \\
\text { zona media } \\
\text { y extremo }\end{array}$ & Total \\
\hline RPI & 0 & 0 & 0 & 5 & 0 & 0 & 1 & 1 & 7 \\
\hline RPA & 9 & 0 & 9 & 8 & 0 & 0 & 5 & 0 & 31 \\
\hline $1-2$ & 4 & 1 & 0 & 2 & 0 & 0 & 0 & 0 & 7 \\
\hline Colado-forjado & 17 & 4 & 15 & 23 & 8 & 3 & 9 & 11 & 90 \\
\hline Equipoise & 5 & 4 & 2 & 1 & 1 & 2 & 2 & 6 & 23 \\
\hline Acker & 1 & 0 & 0 & 0 & 0 & 0 & 0 & 0 & 1 \\
\hline Gillette & 9 & 0 & 0 & 0 & 0 & 0 & 0 & 0 & 9 \\
\hline Total & 28 & 7 & 21 & 56 & 4 & 7 & 25 & 20 & 168 \\
\hline$*$ p=0,0001 & & & & & & & & & \\
\hline
\end{tabular}

bilizador no se observó diferencia estadísticamente significativa ( $p=0,338)$; mientras que en la evaluación de la presencia de contacto con tope se observó diferencia $(p=0,010)$.

\section{DISCUSIÓN}

En la evaluación del adaptado de los retenedores directos se observa que las zonas con mayor porcentaje de adaptación o contacto son las correspondientes al extremo del elemento retentivo y el elemento estabilizador, resultado que coincide con lo observado por Dunham et al. (5). Otras zonas de los elementos retentivo y estabilizador (parte media y unión con el conector menor) y el elemento de soporte (tope), presentan porcentajes de adaptación por debajo del $50 \%$, valores inferiores a lo observado por Dunham et al. (5), por lo que el requisito de soporte y estabilidad del retenedor directo se ve comprometido en una proporción alta de los casos. Estas deficiencias pudieran estar asociadas con las deficiencias observadas previamente en el estudio de evaluación de la aceptación clínica de DPR, realizado en la misma población, donde se evidenció que los parámetro de soporte y retención fueron deficientes en un porcentaje alto de los casos estudiados (9).

Sato et al. (10) describen mediante una evaluación sistemática las causas de las fallas en el apoyo de los ganchos. Refieren que el apoyo no puede ser ajustado en la mayoría de los casos, contrario al ajuste de la retención que se puede realizar en la gran parte de las situaciones. Otro factor que se debe considerar en el adaptado de los retenedores directos según lo reportado por Ahmad et al. (11) es que los planos guías ofrecen cierta resistencia por fricción al desalojo por lo cual estos deben ser considerados. Igualmente en DPR a extensión distal se debe tomar en cuenta la realización del ajuste fisiológico de los componentes del retenedor involucrados en el control de las tensiones que se generan sobre el diente (12). En este aspecto, Carr et al. (8) establecen que los factores que influyen sobre el funcionamiento del retenedor directo son: la localización de los apoyos, el diseño de los conectores menores, su relación con los correspondientes planos guías y la localización de los brazos retentivos. 
En la evaluación específica del elemento de soporte (tope) se observó que menos del $10 \%$ posee contacto en su parte central, resultado inferior a lo observado por otros autores que reportan la presencia de contacto en el $24 \%$ de los casos (5). Sin embargo, el espacio entre el tope y descanso fue similar al reportado previamente por Dunham (11). El mayor porcentaje de los casos presentó contacto solo en el borde del tope, lo que puede generar una apreciación poco exacta del adaptado.

En cuanto a la evaluación del ajuste de la estructura, se ha reportado que el empleo de materiales para detectar contactos, como pasta blanca de pulir, rojo inglés con cloroformo o indicador en spray, presentan limitaciones para evaluar el asentamiento de la estructura debido a que indica la presencia de contacto pero no la magnitud del mismo y se dedica mucho tiempo identificando contactos de punto sin saber realmente cuánto ajuste se requiere. Ante esta situación se recomienda la utilización de un material de mayor espesor como la cera de oclusión blanca, durante la prueba, cuando se ve un color gris existe un pequeño espacio entre el colado y la superficie del diente y cuando la cera permanece blanca, existe un espacio importante entre el colado y el diente. Estas diferencias permiten al operador observar el grado de contacto y obtener una evaluación tridimensional del ajuste. Igualmente la aplicación de cera es útil para identificar contactos en otras áreas del retenedor directo (gancho) cuando la estructura bascula o no asienta completamente después de ajustar los topes (13). En el procedimiento de ajuste del apoyo, es importante considerar que otros elementos de la estructura ubicados por encima del máximo contorno ofrecen soporte a la DPR (14).

Las diferencias estadísticamente significativas observadas en el adaptado del elemento retentivo y estabilizador de los diferentes diseños de retenedores directos empleados en DPR a extensión distal es un resultado predecible debido a las diferencias estructurales y las complicaciones técnicas propias de cada diseño de retenedor. Sin embargo y dentro de las limitaciones de este estudio, se observó que los diseños de retenedores empleados con mayor frecuencia RPA y Colado-Forjado no presentan diferencias estadísticamente significativas en cuanto al adapta- do del elemento retentivo y estabilizador, contrario a lo esperado según lo reportado por Carr et al. (7) relacionado con las desventajas del retenedor Colado-Forjado. Los retenedores tipo Colado-Forjado y RPA fueron frecuentemente usados en los dientes pilares de DPR a extensión distal, resultados similares a lo observado en estudios previos $(4,7)$.

Ante esta situación es importante considerar lo observado por Itoh et al. (15), quienes estudiaron in vitro ambos retenedores y observaron que el empleo del retenedor rígido disminuye la cantidad de movimiento que se genera sobre el diente pilar. No obstante, es mediante estudios clínicos longitudinales controlados con los que se pueden obtener una mejor evidencia para la toma de decisiones en el diseño de DPR.

\section{BIBLIOGRAFÍA}

1. Rudd RW, Bange AA, Rudd KD, Montalvo R. Preparing teeth to receive a removable partial denture. J Prosthet Dent 1999;82(5):536-49.

2. Frank RP, Brudvik JS, Leroux B, Milgrom P, Hawkins N. Relationship between the standards of removable partial denture construction, clinical acceptability and patient satisfaction. J Prosthet Dent 2000;83(5):521-7.

3. Sato Y. Clinical methods for adjusting retention force of cast claps. J Prosthet Dent 1999;82(5): 557-61.

4. Sánchez AE, Morelly E, Jorge V. Evaluación de las estructuras metálicas de los casos de Dentaduras Parciales Removibles de la Facultad de Odontología de la Universidad Central de Venezuela. Acta Odont Venez 2007;45(3):388-93.

5. Dunham D, Brudvik JS, Morris WJ, Plummer KD, Cameron SM. A clinical investigation of the fit of removable partial dental prosthesis clasp assemblies. J Prosthet Dent 2006; 95(4):323-6.

6. Sánchez AE, Tarantini M. Frecuencia de los tipos de maxilares parcialmente edéntulos y diseños indicados para su tratamiento con prótesis par- 
ciales removibles en la UCV. Acta Odont Venez 1993;31(2):27-38.

7. Sánchez AE, Troconis I, Morelly E. La prótesis parcial removible en la práctica odontológica de Caracas, Venezuela. Acta Odont Venez 1999;37: 123-35.

8. Carr A, McGivney G, Brow D. Mc Cracken prótesis parcial removable. $11^{\mathrm{a}}$ ed Elsevier: Madrid España; 2006.

9. Sánchez AE. Aceptación clínica de Dentaduras Parciales Removibles en sujetos tratados en la Facultad de Odontología de la Universidad Central de Venezuela. Acta Odont Venez 2011;49(3) [aceptado para publicación].

10. Sato Y, Shimodaira O, Kitagawa N. Systematic clinical evaluation and correction procedures for support of Removable Partial Dentures. J Prosthodont 2008;17(3):228-32.

11. Ahmad I, Sherrif M, Waters N. The effect of reducing the number of clasps on removable partial denture retention. J Prosthet Dent 1992;68(6):928-33.

12. Donahue T. Factors that augment the role of direct retainers in mandibular distal-extension removable partial dentures. J Prosthet Dent 1988; 60(6):696-9.

13. Rudd RW, Rudd KD. A review of 243 errors possible during the fabrication of a removable partial denture: Part II. J Prosthet Dent. 2001;86 (3):262-76.

14. Brudvik J. Advanced removable partial dentures. Editorial Quintessence: Chicago; 1999.

15. Itoh H, Baba K, Aridome K, Okada D, Tokuda A, Nishiyama A, Miura H, Igarashi Y. Effect of direct retainer and major connector designs on RPD and abutment tooth movement dynamics. J Oral Rehabil 2008;35(11):810-5.

\section{CORRESPONDENCIA}

Andrés Eloy Sánchez Y.

Cátedra de Dentaduras Parciales Removibles, piso 6. Facultad de Odontología. Universidad Central de Venezuela.

Avenida Los Ilustres.

Los Chaguaramos.

Caracas 1053. Venezuela.

Correo electrónico: drandreseloy@hotmail.com 